\title{
Study of the corrective forces applied by a dynamic derotation brace (DDB)
}

\author{
I Loukos ${ }^{*}$, C Nicolopoulos $^{2}$, C Zachariou $^{3}$ \\ From 8th International Conference on Conservative Management of Spinal Deformities and SOSORT 2011 \\ Annual Meeting \\ Barcelona, Spain. 19-21 May 2011
}

\section{Background and purpose}

The direct forces exerted by the brace pad were analysed, on 44 idiopathic scoliotic patients (38 girls, 6 boys) treated with a DDB [1]. Twenty seven patients had a single right thoracic curve and 17 had a single left or right thoracolumbar curve. The aim was the analysis of the corrective forces, exerted at the skin-brace interface, by altering the posture, activity and strap tension [2-7].

\section{Materials and methods}

We used the F-Socket 9801 pressure sensor and the MatScan Research BETA STAM v. 6.30 software (TekScan, Boston MA, USA), and measurements were taken in nine body postures. The patients were divided into three groups: those who were wearing for first time the brace, those who changed their brace with a new one and those who made corrections on their brace.

\section{Results}

Changes in strap tension, body posture, resulted in statistically significant alterations of the interface pressure on the pads and thus of the resultant forces exerted on the patient's body by the pads. Comparing the three groups in relation with the magnitude of the mean exerted force, the correction of the brace caused the highest mean exerted force.

\section{Conclusions}

Even though the TekScan system does not provide direct information on the correction of spinal curvature, it appears to be a useful tool in the treatment of scoliotic patients. Also, the analysis of the corrective forces seems to be very helpful trying to achieve brace's optimal fit and the same time the best therapeutic result for the patient.

\section{Author details}

'Medical School, National \& Capodistrian University of Athens Anoixi, Greece. ${ }^{2}$ ORTHO-FOOT Center, Athens, Greece. ${ }^{3}$ KAT Hospital of Athens, Greece.

Published: 27 January 2012

\section{References}

1. Grivas TB, Bountis A, Vrasami I, Bardakos NV: Brace technology thematic series: the dynamic derotation brace. Scoliosis 2010, 5:20.

2. Aubin CE, Labelle $H$, Ruszkowski $A$, et al: Variability of strap tension in brace treatment for adolescent idiopathic scoliosis. Spine 1999, 24(4):349-54.

3. Mac-Thiong $J M$, Petit $Y$, Aubin $C E$, et al: Biomechanical evaluation of the Boston brace system for the treatment of adolescent idiopathic scoliosis. Spine 2003, 29(1):26-32.

4. Nicolopoulos CS, Anderson EG, Solomonidis SE, et al: Evaluation of the gait analysis F-Scan pressure system: clinical tool or toy? The Foot 2000, 10:124-130.

5. Pham VM, Houilliez A, Schill A, et al: Study of the pressures applied by a Chêneau brace for correction of adolescent idiopathic scoliosis. Prosthet Orthot Int 2008, 32(3):345-355.

6. van den Hout JAAM, van Rhijn LW, van den Munckhof RJH, et al: Interface corrective force measurements in Boston brace treatment. Eur Spine J 2002, 11:332-335.

7. Wong MS, Evans JH: Biomechanical evaluation of the Milwaukee brace. Prosthet Orthot Int 1998, 22:54-67.

doi:10.1186/1748-7161-7-S1-031

Cite this article as: Loukos et al: Study of the corrective forces applied by a dynamic derotation brace (DDB). Scoliosis 2012 7(Suppl 1):031. 